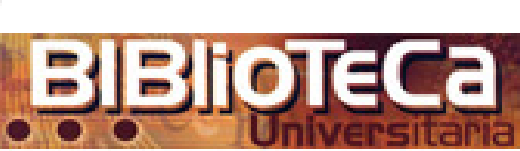

Biblioteca Universitaria

ISSN: 0187-750X

public@dgb.unam.mx

Universidad Nacional Autónoma de México

México

Rodríguez García, Ariel Alejandro; González Castillo, Raúl Ariel Las normas mexicanas de catalogación de acervos videográficos y documentos fonográficos, ¿Visión a corto o largo plazo?

Biblioteca Universitaria, vol. 14, núm. 1, enero-junio, 2011, pp. 3-17

Universidad Nacional Autónoma de México

Distrito Federal, México

Disponible en: http://www.redalyc.org/articulo.oa?id=28521141002

- Cómo citar el artículo

- Número completo

- Más información del artículo

Página de la revista en redalyc.org

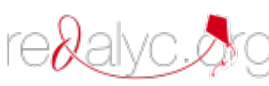

Sistema de Información Científica

Red de Revistas Científicas de América Latina, el Caribe, España y Portugal Proyecto académico sin fines de lucro, desarrollado bajo la iniciativa de acceso abierto 


\title{
Las normas mexicanas de catalogación de acervos videográficos y documentos fonográficos, ¿visión a corto o largo plazo?
}

\author{
Mexican Cataloging Standards for Videographic and Phonographic \\ Documents, a Short or Long Term Vision?
}

Ariel Alejandro Rodríguez García", Raúl Ariel González Castillo**

\section{Resulnen}

En este trabajo se hace un estudio comparativo entre las normas mexicanas de catalogación de acervos videográficos y de documentos fonográficos y las Reglas de Catalogación Angloamericanas, segunda edición, en particular los capítulos seis (videograbaciones) y siete (grabaciones sonoras). Lo anterior con el fin de observar si las normas mexicanas proporcionan consistencia en la presentación de los datos bibliográficos y la asignación del punto de acceso, asuntos que son considerados como los criterios más importantes en el control de calidad de la catalogación. Un argumento importante del estudio es que tanto las instrucciones como la estructura de ambas normas mexicanas están muy lejos de ser consideradas como referentes en el proceso de catalogación en las instituciones nacionales. Además hace falta consenso en el país para que las normas mexicanas sean tomadas en cuenta, puesto que su uso y aplicación está en riesgo de apartarse de una tradición reconocida y compartida.

PALABRAS ClAVE: catalogación descriptiva, normas mexicanas de catalogación, RCAA2

\section{Abstract}

In this work a comparative study of the Mexican standards for cataloging videographic and phonographic documents and the Anglo-American Cataloguing Rules, Second edition, is made in particular for Chapters Six (video recordings) and Seven (sound recordings). This to see if the Mexican standards provide consistency in the presentation of bibliographic data and access point allocation, issues that are considered the most important criteria in the quality control of cataloging. An important argument of this study is that both the instructions and the structure of both Mexican standards are far from being considered as a reference point in the cataloging process in national institutions. There is also a lack of consensus in this country, so Mexican standards be taken into account, since their use and application is at risk of departing from a recognized and shared tradition.

KEYwords: Descriptive cataloging, Mexican cataloging standards, RCAA2

* Centro Universitario de Investigaciones Bibliotecológicas, Torre II Humanidades piso 12, UNAM, C.U., C.P. 04510, México, D.F. México. Correo electrónico: ariel@cuib.unam.mx.

** Biblioteca de la Escuela Nacional de Pintura, Escultura y Grabado "La Esmeralda". Centro Nacional de las Artes, Av. Río Churubusco No. 79, esq. Calz. Tlalpan, C.P. 04220 Col. Country Club, Coyoacán, México, D. F. México. Correo electrónico: raulgc@unam.mx 


\section{Introducción}

$H$

oy, al estudiar el campo de la catalogación podemos reconocer nuevas definiciones y conceptualizaciones que revelan confrontaciones profundas en torno a las prácticas de la identificación, descripción y transcripción bibliográfica, inscriptas sin lugar a dudas en el terreno de la organización de la información.

Nuestro punto de partida es entender que los criterios más importantes en el control de calidad de la catalogación son la presentación de los datos bibliográficos y la asignación de un punto de acceso, los cuales son praxis basadas en decisiones tomadas de una serie de normas bibliotecarias como son las Reglas de Catalogación Angloamericanas, segunda edición, las normas internacionales para la descripción bibliográfica y otras más empleadas para crear los registros bibliográficos o de autoridad.

Desde esta conceptualización, se trata de revisar comparativamente las normas mexicanas de catalogación de acervos videográficos $y$ de documentos fonográficos $y$ las Reglas de Catalogación Angloamericanas, segunda edición, con el firme propósito de evaluar si las normas mexicanas se benefician de los cambios ocurridos en el plano internacional de la catalogación o su enfoque beneficia a las instituciones nacionales en las tareas de obtener, procesar e intercambiar información, funciones principales del control bibliográfico. ${ }^{1}$ Este punto de partida puede, para algunos, entenderse como el más común y divulgado en la generalización de estudios comparados que denuncian el buen o mal uso de las reglas de catalogación. Puede entenderse, también, como la forma de salvar los seudo olvidos al crear normas de aplicación nacional, como es el caso que estudiamos aquí. Tratamos, en cambio, de superar estas antípodas recuperando las propuestas críticas, alimentando nuestros planteamientos de catalogación con investigaciones científicas en el campo de las tecnologías de la información, la teoría de sistemas y los mismos fundamentos de la organización de la información, intentando responder la perspectiva a corto o largo plazo de las actuales normas mexicanas de catalogación.

GARDUÑo Vera, Roberto. Modelo bibliográfico basado en formatos de intercambio y en normas, p.28.
En este trabajo se presentan algunos de los avances que se tienen de la investigación mayor relacionada con el proyecto Impacto del FRBR en la normativa de la descripción bibliográfica, que se lleva a cabo en el Centro Universitario de Investigaciones Bibliotecológicas. ${ }^{2}$

\section{Panorama actual de la normativa internacional para la descripción bibliográfica}

En los últimos trece años (1997-2010), el problema central que se ha tratado en la normativa internacional para la descripción bibliográfica ha sido la incorporación de los modelos conceptuales entidad-relación, como los Requerimientos Funcionales para los Registros Bibliográficos (FRBR por sus siglas en inglés), las limitaciones en la estructura lógica de las Reglas de Catalogación Angloamericanas, segunda edición, así como la aparición de los recursos de información digital, entre otros más, y la reciente liberación de los lineamientos para la Descripción y Acceso a Recursos.

De acuerdo con lo anterior, las preguntas que servirán de guía para nuestra lectura sobre la normativa para la descripción bibliográfica son las siguientes: ¿̇cómo se constituyeron las normas mexicanas de catalogación?, ¿cuáles han sido los mecanismos que se han desplegado para aceptar, aplicar, evaluar y usar estas normas principalmente en fonotecas y videotecas nacionales?, al momento de transferir los datos descriptivos de las videograbaciones y grabaciones sonoras ¿qué tipo de registro bibliográfico se produce?, y, ¿qué prácticas de catalogación se han consolidado con el uso de las normas mexicanas de catalogación?

El corpus teórico de nuestra investigación se sustenta en la revisión de los avances que se han venido dando desde 1997 en toda la teoría de la catalogación y la creación de las estructuras de metadatos para recursos de información digital tanto de audio como de video. En cuanto a los progresos de la teoría de la

2 Universidad Nacional Autónoma de México. Centro Universitario de Investigaciones Bibliotecológicas. Informe de actividades 2009-2010 [en línea]. Jaime Ríos Ortega. <http://cuib. unam.mx/f//nforme-2009-2010.pdf> 


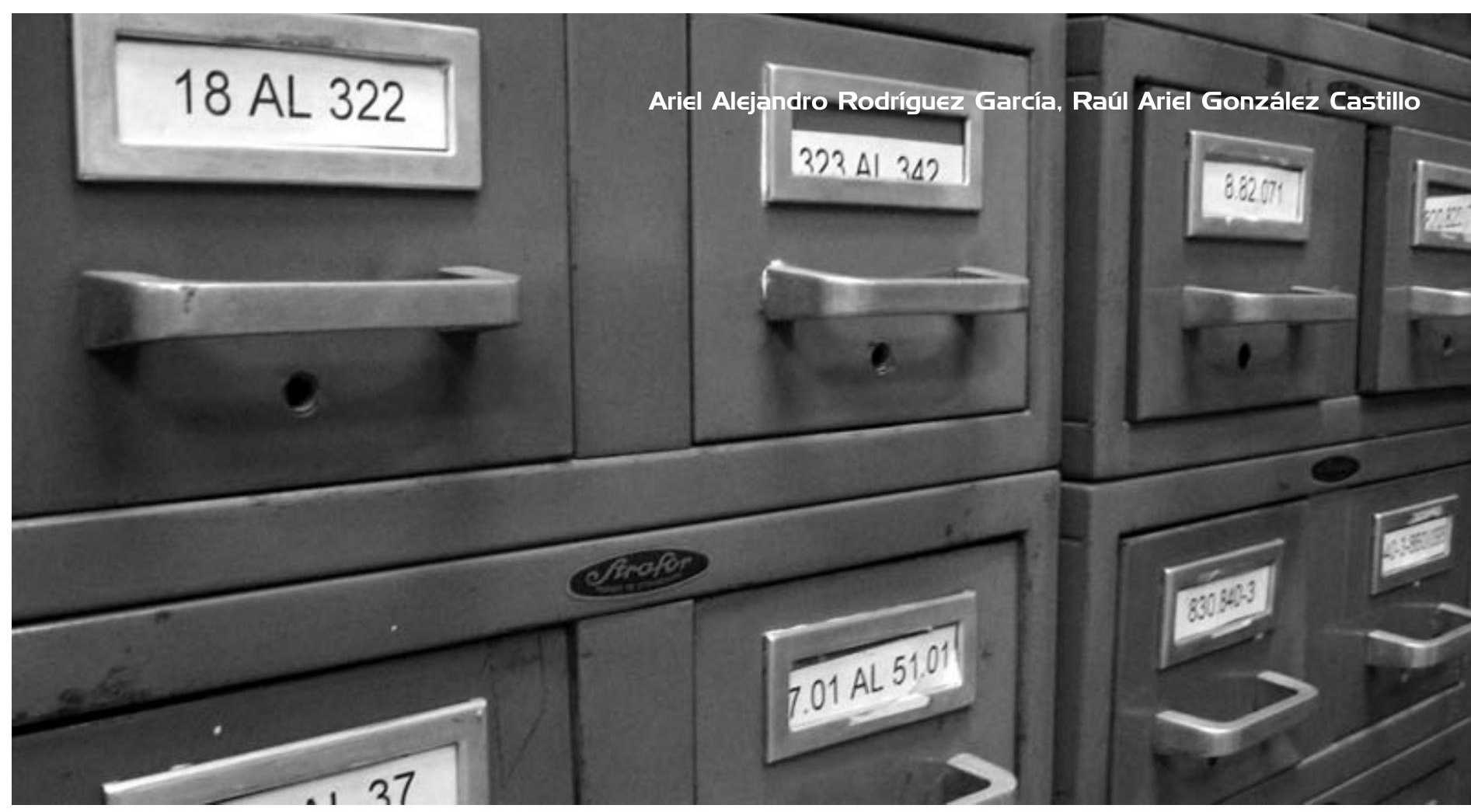

catalogación es evidente que se ha dado un giro, y de manera sintética podríamos afirmar que se ha optado por incorporar los principios de la teoría de sistemas, el análisis de las bases de datos y el modelado de la entidad-relación a toda la normativa. ${ }^{3}$ Solamente falta que al aplicar los principios internacionales de catalogación, las normas internacionales de descripción bibliográficas y los lineamientos para la descripción y acceso a recursos se pueda observar la catalogación desde una perspectiva particular.

Frente a la crisis que se veía venir en los años noventa en la catalogación ${ }^{4}$ se hizo necesaria una reforma que asegurara un proceso de catalogación de calidad. La función de la práctica de la catalogación ${ }^{5}$ es organizar una colección de ítems bibliográficos para facilitar su identificación, localización, acceso y uso, y por supuesto

3 IFLA Study Group on the Functional Requirements for Bibliographic Records. Functional Requirements for Bibliographic Records: final report. Approved by the Standing Committee of the IFLA Section on Cataloging. September 1997, as amended and corrected through February 2008. [en línea]. <http// www.ifla.org/VII/s13/frbr/index.htm>, h. 10.

4 López Guillamón, Ignacio. Evolución reciente de la catalogación. Anales de documentación, p.141-152.

5 LeVy, David M. "Cataloging in the digital order" [en línea]. Digital libraries $95<$ http://csdl.tamu.edu/DL95/papers/levy/levy.html> que el principal método para organizar grandes colecciones es desarrollar un catálogo, el cual consiste en un conjunto de entradas, cada una de las cuales muestra un ítem y sus características como autor, título, editor, temas, entre otros; el catálogo en sí mismo es una colección de registros que están arreglados. La catalogación con calidad se constituye como un nuevo discurso entre los bibliotecarios, la cual explora sus respuestas en la reciente idea de la racionalidad técnica; es decir, la transformación del proceso de catalogación con base en el uso de las actuales tecnologías de la información y sistemas de recuperación de información.

Sobre los requerimientos funcionales para los registros bibliográficos ${ }^{6}$ la constante que se ha encontrado ha sido la falta de entendimiento de las entidades, los atributos y las relaciones; del enfoque de sistemas aplicado a la solución de los problemas de catalogación; de los cambios en el sentido, uso y aplicación de la ter-

6 En la revista Library resources \& technical services, july 2000, vol. 44, num.33 se publican diversos artículos respecto a la perspectiva de uso y aplicación del modelo FRBR. En el 2005 se publica Le Bouef, Patrick. Functional requirements for bibliographic records (FRBR): hype or cure-all? New York: The Haworth Information, 2005. Para 2007 Arlene Taylor edita Understanding FRBR: what it is and how it will affect our retrieval tools. Arlene Taylor, ed. Westport, Connecticut. Libraries Unlimited. 2007. 192 p. 


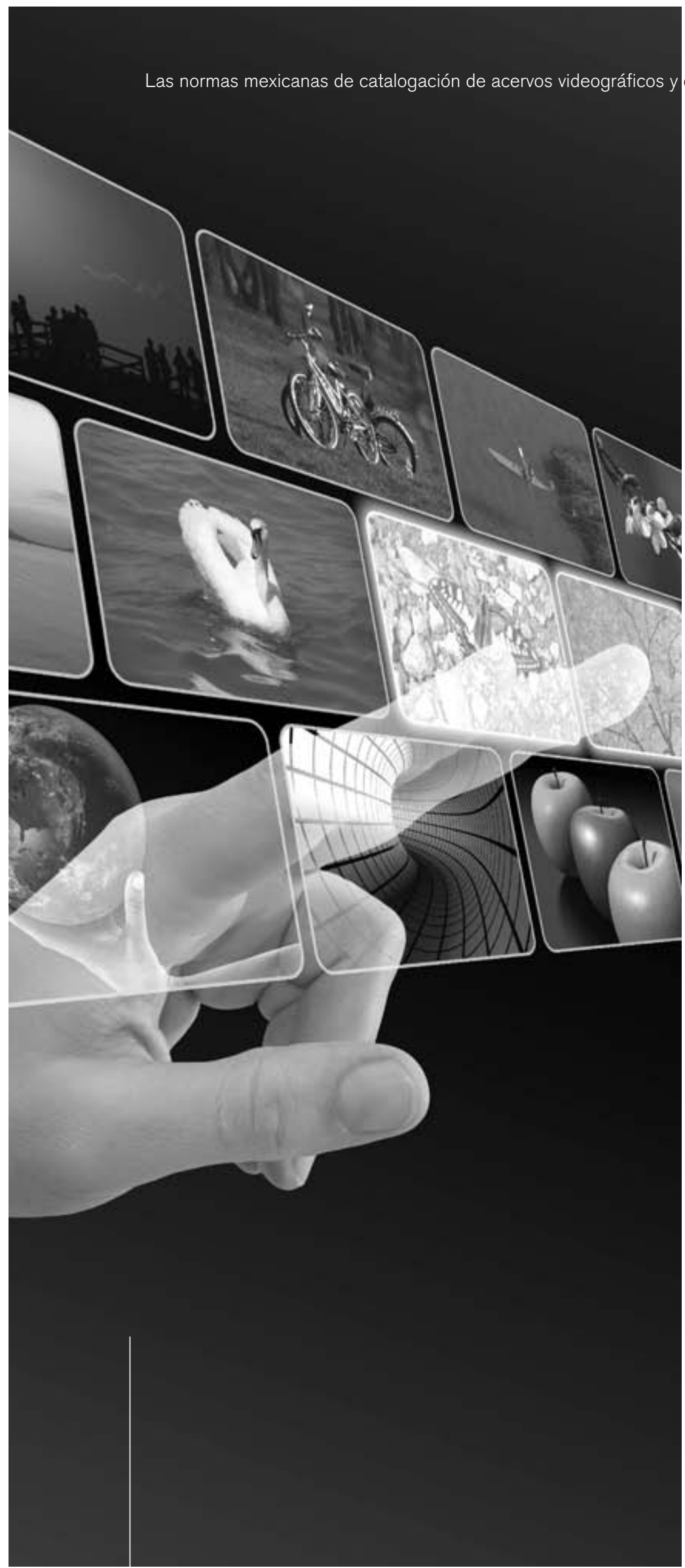

documentos fonográficos, ęvisión a corto o largo plazo?

minología que se desarrolla en el modelo, así como de la orientación que se tiene respecto a las necesidades e interés del usuario al momento que se despliegan los datos de las entidades y relaciones bibliográficas.

Como lo señalan Bianchini y Guerrini7, hoy tenemos a disposición los principios y estándares de descripción bibliográfica; ambos constituyen un patrimonio para la catalogación reconocido a nivel internacional. Las variaciones locales se permiten, por supuesto, pero en la actualidad el recurso de la elección de ámbito local implica el riesgo de apartarse de una tradición reconocida y compartida.

En cuanto a los lineamientos para la descripción y acceso a recursos, Oliver ${ }^{8}$ menciona que pretenden ejemplificar, en forma lo más adecuadamente sistematizada, el proceso de catalogación; es decir, qué necesidades de información deben registrarse y cómo éstas deberán registrarse para auxiliar al usuario en la navegación a lo largo de las bases de datos o catálogos y ellos puedan encontrar, identificar, seleccionar u obtener un recurso que sea apropiado a sus necesidades de información.

Después de haber revisado los aspectos del nuevo orden en la práctica de la catalogación y las modificaciones que se han hecho a las distintas normativas para la descripción bibliográfica, observaremos concisamente qué está sucediendo con el desarrollo de los catálogos altamente sofisticados y sistemáticos que han adoptado procedimientos de la nueva era de la catalogación, es decir, aquellos que están sustentados en la tecnología de la información y comunicación.

La incorporación de esta tecnología es una exigencia para los actuales catálogos en línea y sistemas de recuperación de información que se encuentran en Internet porque de aquella depende la creación de nuevos productos y servicios orientados a los usuarios que buscan, acceden y recuperan información. En este sentido, la intervención de la tecnología con las necesidades de información del usuario no puede manejarse como una relación externa

BianchinI, Carlo y GuerrinI, Mauro. Quis custodiet ipsos custodies? Observaciones sobre la relación entre FRBR, ICP, ISBD Y RDA. Anales de documentación, p. 321.

Oliver, Chris. Changing to RdA. Feliciter, p. 251. 
"experto-usuario" sino que en esta relación se está enfatizando la creación de un proceso de aprendizaje mutuo, para lograr soluciones más eficientes y efectivas.

Emanuel $^{9}$ señala que la siguiente generación de catálogos se obligará a encontrar nuevos mecanismos de búsqueda de información para actividades específicas. Es decir, su arreglo no se limitará a proporcionar un solo tipo de despliegue, sino que deberá enfatizar el lugar específico del recurso y a partir de éste permitir la redefinición de la búsqueda, hasta que la petición del usuario encuentre la información que necesita.

La relación entre nuevos catálogos, normas bibliográficas y usuarios es, entonces, una relación cargada hacia el despliegue e interface de acceso a la información. Entre estos elementos se libra una lucha. En la historia del catálogo, esa lucha tomó características diferentes y fue definida como un desacuerdo entre lo que se representa y describe por el bibliotecario y lo que busca el usuario.

El movimiento de los "catálogos nextgen" es otro aspecto sobre cómo los bibliotecarios están adaptándose a los cambios en la era digital. Los catálogos en línea cambian lentamente debido a su natural constitución, pero los cambios se perciben con la incorporación de los nuevos patrones de búsqueda y los servicios y productos de valor agregado para el usuario.

Entonces, una de las fases del proceso de catalogación consiste en provocar ciertos desequilibrios intencionados e intervenir en el proceso de reequilibración, para favorecer o mediar entre el catálogo y el usuario de la información.

En la práctica de la catalogación constantemente tiene lugar una negociación entre la normativa y el juicio del catalogador $^{10}$, en la que este último "interpreta, apli-

9 EmANuEL, Jenny. Next generation catalogs: what do they do and why should we care? Reference \& user Services Quarterly, p. 118.

10 Rodríguez García, Ariel Alejandro. "El criterio del catalogador

y la nueva normativa para la descripción y acceso" [en línea]. En: Memorias del VI Seminario Hispano-Mexicano de Investigación en bibliotecología y documentación. Boletín ANABAD, abril-junio, 2009, no. 2, p. 247-258. <http://www.anabad.org/ boletin/index.php?id $=168 \& c=1>$ ca y decide" qué regla será aplicada en la elaboración del registro descriptivo del ejemplar que se tiene en la mano y así cumplir con los objetivos planteados por Cutter ${ }^{11}$ para la creación del catálogo.

Lo anterior significa que habrá diferentes niveles de descripción y utilización de los datos bibliográficos, pero siempre buscando las ventajas de recurrir a las estructuras de búsqueda y recuperación de la información en las bases de datos.

\section{Las normas mexicanas de catalogación}

Si el panorama internacional en la descripción bibliográfica ha estado en constante evolución en los últimos tres lustros, es conveniente que hagamos una revisión respecto a las nociones fundamentales en las que se basan las normas mexicanas de catalogación de acervos videográficos ${ }^{12}$ (NMX-R-001-SCFI-2007) y documentos fonográficos ${ }^{13}$ (NMX-R-002-SCFI-2009).

Las normas mexicanas de catalogación se conciben a sí mismas como las pautas a seguir por aquellas instituciones del país que cuenten con acervos videográficos y fonográficos porque las normativas internacionales existentes para la descripción bibliográfica-archivística y las desarrolladas por la Federación Internacional de Archivos de Televisión (IFTA por sus siglas en inglés) y la Asociación de Archivos Sonoros y Audiovisuales (IASA por sus siglas en inglés) no han resuelto los problemas de almacenamiento, descripción, representación e intercambio de información de los acervos nacionales.

11 Rules for a dictionary catalog: selection. Reprinted from Charles A. Cutter. Rules for a dictionary catalog, 4th ed. (Washington, D.C.: Government Printing Office, 1094.) En: Foundations of cataloging: a sourcebook. Michael Carpenter, Elaine Svenonius, editors, p. 65.

12 Norma Mexicana de Catalogación de Acervos Videográficos NMX-R-001-SCFI-2007.

13 Norma mexicana NMX-R-002-SCFI-2009, documentos fonográficos [en línea]: lineamientos para su catalogación. Comité Técnico de Normalización Nacional de Documentación. México: Secretaría de Economía. 2010. <http://200.77.231.100/ work/normas/nmx/2010/nmx-r-002-scfi-2009.pdf> 


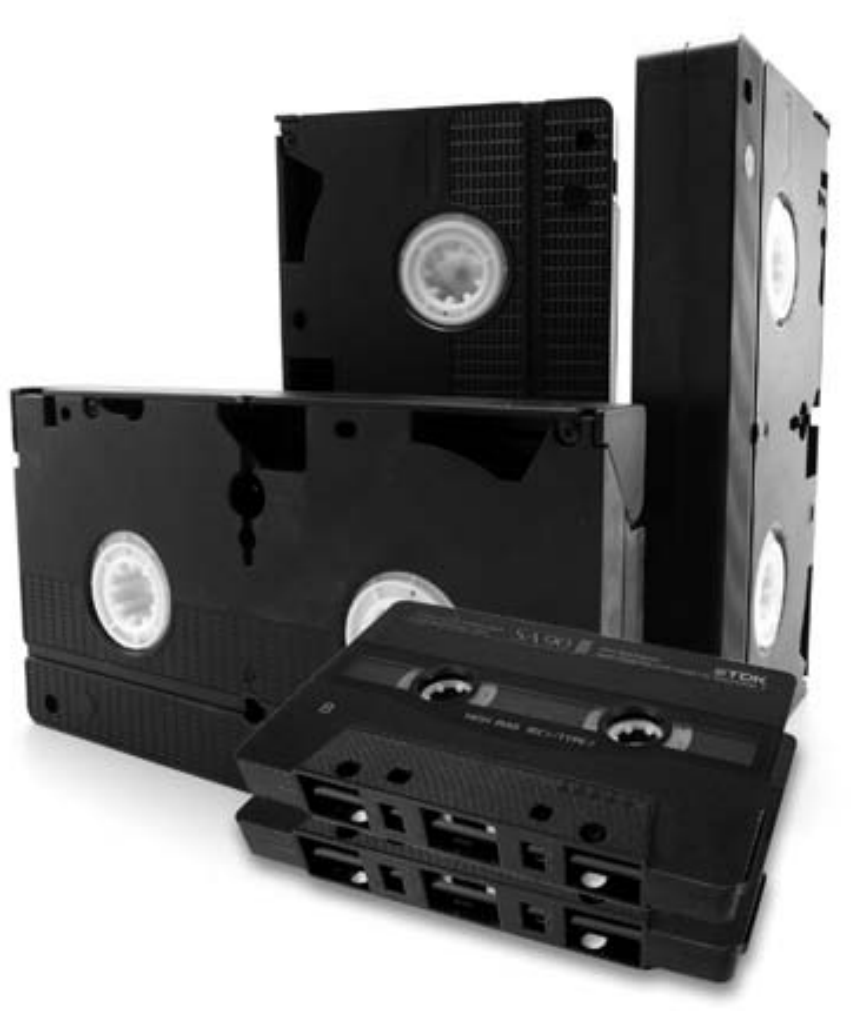

Por lo que se refiere a la elaboración y constitución de las normas mexicanas, diversas instituciones convocadas inicialmente por la Dirección General de Televisión Educativa de la Secretaría de Educación Pública, luego por la Dirección General de Normas de la entonces SECOFI, se constituyen como el Comité Técnico de Normalización Nacional de Documentación (COTENNDOC), quien será el responsable de iniciar los trabajos de creación de las normas. La norma mexicana de catalogación de acervos videográficos, primera en desarrollarse, se da a conocer a través del Diario Oficial de la Federación el 18 de diciembre de 2000.

En cuanto a la norma mexicana para documentos fonográficos-lineamientos para su catalogación, se sabe que sus antecedentes datan del año 2001 cuando surge a través de la cédula mínima de datos, elaborada por el Seminario Permanente de Fonotecas. En el 2002, el COTENNDOC inicia el proyecto para elaborar una norma mexicana que sirviese para describir los acervos sonoros nacionales. Al siguiente año (2003) surge el Pro- yecto de Catalogación Descriptiva denominado Base de Creación para la Norma Mexicana de Catalogación Sonora con la participación de varias instituciones convocadas por Radio Educación. Como resultado de este trabajo conjunto se anunció la Norma Mexicana de Catalogación de Documentos Fonográficos, basada en el estudio de normas internacionales de catalogación, así como la Guía para la Redacción, Estructuración y Presentación de las Normas Mexicanas (NMX-Z013/1-1997) basada en la Iso Guide for the Presentation of International Standards and Technical Reports.

La versión más reciente de la norma documentos fonográficos-lineamientos para su catalogación ${ }^{14}$ es la NMX-R-002-SCFI-2009, cuya declaratoria de aprobación se manifiesta en el Diario Oficial de la Federación del 8 de marzo del 2010.

Ambas normas mexicanas de catalogación tienen la intención de orientar el desarrollo de los registros descriptivos y la creación de un medio de recuperación de información para las videograbaciones y documentos fonográficos que se encuentran en los acervos de instituciones como las radiodifusoras y televisoras del país. Pero no obstante, habría que hacer una revisión general a sus estructuras con el fin de identificar las áreas de descripción y elementos ${ }^{15}$ que permiten la uniformidad en los registros, como está señalado en las Normas Internacionales para Descripción Bibliográfica.

En la siguiente tabla se resume el contenido de ambas normas y contiene su campo de aplicación, las áreas de descripción, así como su correspondencia con normas internacionales.

14 Comité Técnico de Normalización Nacional de Documentación [en línea]. <http://www.cotenndoc.org.mx/> [Consulta: 1 julio 2010].

15 De acuerdo con las ISBD (G) un área es la sección de la descripción bibliográfica que comprende datos de una categoría o un conjunto de categorías. La descripción bibliográfica es el conjunto de datos bibliográficos que describen e identifican un documento En tanto que los elementos son palabras o frases o grupo de caracteres que representa una unidad de información bibliográfica diferenciada y que forma parte de un área de la descripción bibliográfica. 


\begin{tabular}{|c|c|c|}
\hline & NMX-R-001-SCFI-2007 & NMX-R-002-SCFI-2009 \\
\hline $\begin{array}{l}\text { Campo de } \\
\text { aplicación }\end{array}$ & $\begin{array}{l}\text { Está orientada preferentemente a la catalogación } \\
\text { de acervos audiovisuales nacionales, educativos } \\
\text { y culturales y a todos aquellos que se compren- } \\
\text { den como patrimonio de interés público. }{ }^{16}\end{array}$ & $\begin{array}{l}\text { Aunque la Norma ha sido concebida para } \\
\text { describir todo tipo de documentos fonográfi- } \\
\text { cos publicados, no publicados y radiofónicos, } \\
\text { contenidos en diversos soportes, se pone } \\
\text { especial énfasis en establecer criterios de } \\
\text { normalización en la catalogación de los do- } \\
\text { cumentos "no publicados" que se encuentran } \\
\text { en los diferentes archivos fonográficos ubica- } \\
\text { dos especialmente en centros de investiga- } \\
\text { ción de diversos caracteres (histórico, etnoló- } \\
\text { gico, antropológico, lingüístico, etc.), escuelas } \\
\text { y radiodifusoras cuya producción regular- } \\
\text { mente no persigue fines comerciales. }{ }^{17}\end{array}$ \\
\hline $\begin{array}{l}\text { Áreas de } \\
\text { descripción }\end{array}$ & $\begin{array}{l}\text { Acervos videográficos } \\
\text { Título } \\
\text { Mención de responsabilidad } \\
\text { Producción } \\
\text { Lugar } \\
\text { Nombre } \\
\text { Fecha } \\
\text { Descripción física } \\
\text { Formato del soporte y extensión } \\
\text { Duración total del soporte } \\
\text { Soporte } \\
\text { Velocidad de grabación/reproducción } \\
\text { Serie o proyecto } \\
\text { Notas } \\
\text { Generales } \\
\text { Resumen } \\
\text { Contenido } \\
\text { Temas/palabras clave } \\
\text { Género } \\
\text { Créditos } \\
\text { Participantes } \\
\text { Duración de la grabación } \\
\text { Idioma }\end{array}$ & $\begin{array}{l}\text { Documentos fonográficos } \\
\text { Título } \\
\text { Identificación } \\
\text { Mención de responsabilidad } \\
\text { Contenido } \\
\text { Versión } \\
\text { Descripción técnica } \\
\text { Disponibilidad } \\
\text { Observaciones } \\
\text { Sección de imágenes de archivo } \\
\text { Título } \\
\text { Identificación } \\
\text { Mención de responsabilidad } \\
\text { Contenido } \\
\text { Descripción técnica } \\
\text { Disponibilidad } \\
\text { Observaciones }\end{array}$ \\
\hline $\begin{array}{l}\text { Concordancia } \\
\text { con normas } \\
\text { internacionales }\end{array}$ & $\begin{array}{l}\text { Esta norma no coincide con alguna norma } \\
\text { internacional, por no existir ninguna sobre el } \\
\text { tema específico tratado. }{ }^{18}\end{array}$ & $\begin{array}{l}\text { Esta norma mexicana no es equivalente a } \\
\text { alguna norma internacional ni nacional por } \\
\text { no existir referencia alguna al momento de } \\
\text { su elaboración. }{ }^{19}\end{array}$ \\
\hline
\end{tabular}

\section{Tabla 1 Estructura de las Normas Mexicanas de Catalogación}

16 Norma Mexicana de Catalogación de Acervos Videográficos NMX-R-001-SCFI-2007, op. cit., p. 19.

17 Norma mexicana NMX-R-002-SCFI-2009, documentos fonográficos [en línea]: lineamientos para su catalogación, op. cit,, p., 18.

18 Norma Mexicana de Catalogación de Acervos Videográficos NMX-R-001-sCFl-2007, op. cit., p. 60.

19 Norma mexicana NMX-R-002-SCFI-2009, documentos fonográficos [en línea]: lineamientos para su catalogación, op. cit,, p.45. 


\section{Las Reglas de Catalogación Angloamericanas, segunda edición}

Si hemos tomado a las Reglas de Catalogación Angloamericanas como referente para explicar la perspectiva de las normas mexicanas es porque resulta fundamental desde el punto de vista bibliotecológico que el paradigma dominante de la descripción bibliográfica, "el ítem que se tiene a la mano", sea el procedimiento cuya aplicación se observa en la presentación de los datos bibliográficos y la asignación de los puntos de acceso, mismos que son considerados como los dos criterios más importantes en el control de calidad de la catalogación.

Otra razón que, confesamos, es más de análisis ideológico concerniente a hacer mención del equívoco frecuente -entre especialistas y no especialistas- de que diferentes procesos son dialógicos, participativos, no autoritarios puesto que contemplan mecanismos de retroalimentación.

Las reglas de catalogación tienen como antecedente los primeros códigos de catalogación elaborados por personajes preocupados por proporcionar un orden lógico a los datos que se extraen de las obras que se encontraban en las colecciones de la biblioteca; después se buscó crear un conjunto de normas que permitieran uniformar y controlar los datos descriptivos y representativos del autor y contenido. Así es como surgen los principios y normas internacionales para la descripción bibliográfica y los códigos de catalogación con las reglas angloamericanas.

Por lo que se refiere a las reglas de catalogación, en la tabla 2 -de manera sintética- podemos observar su constitución y estructura.

Para el análisis que se está haciendo de las normas mexicanas en contraste con las reglas de catalogación, es valioso destacar la estructura y orden de la Parte I de las reglas, ya que ahí se pueden observar algunas semejanzas y diferencias.

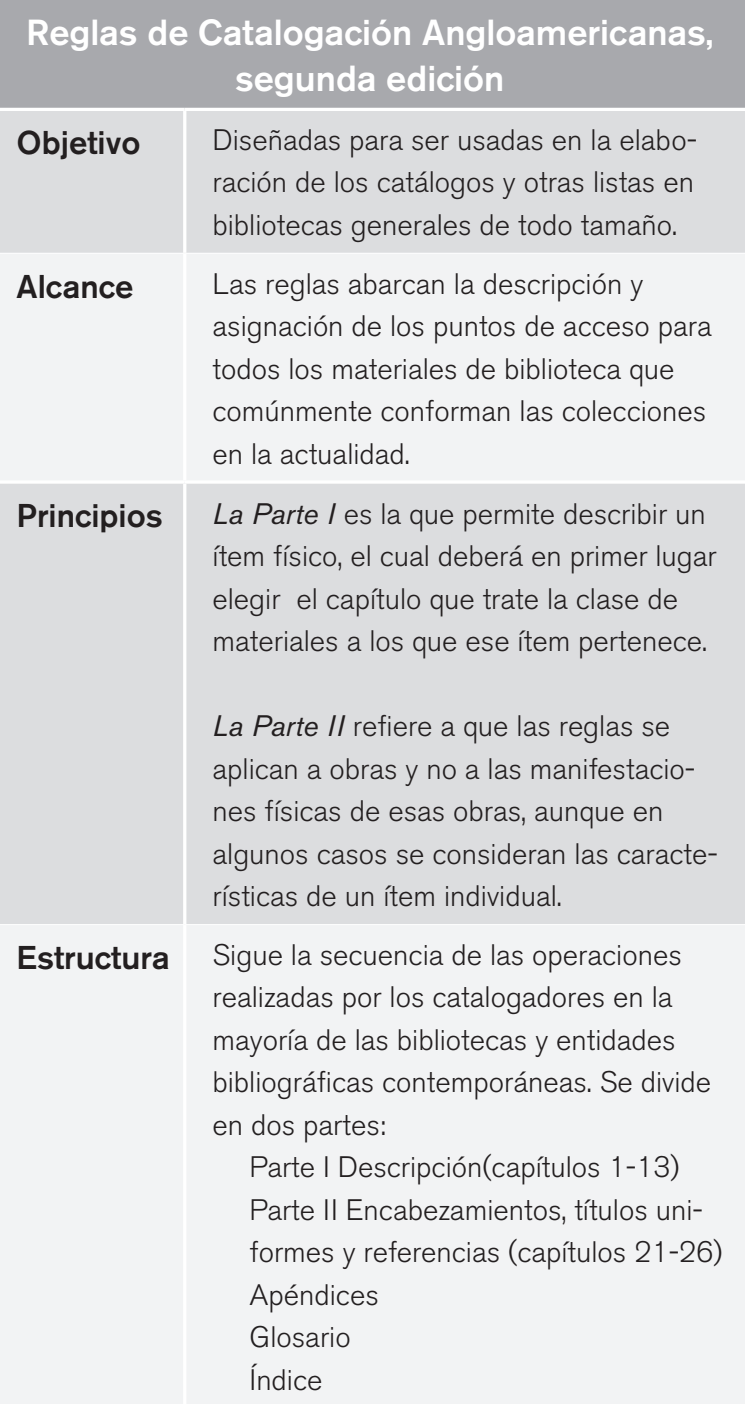

Tabla 2 Estructura de las Reglas de Catalogación

De acuerdo con la regla 0.3 que a la letra señala:

"Las reglas siguen una secuencia de las operaciones realizadas por los catalogadores en la mayoría de las bibliotecas y entidades bibliográficas contemporáneas. La Parte I trata de las instrucciones relativas a la información que describe el ítem que se cataloga y la Parte II trata de la determinación y establecimiento de los encabezamientos (puntos de acceso) bajo los cuales la información descriptiva se presenta a los usuarios del catálogo; y de la elaboración de las refe- 
rencias que se hacen a esos encabezamientos. Las instrucciones propias de las Partes I y II comienzan en Parte I-1 y en la Parte II-1, respectivamente."20

Es preciso señalar que las reglas de catalogación van de lo general a lo específico, de ahí que la Parte I se refiera al medio físico del ítem que se cataloga y la Parte II a los tipos de asientos principal y secundarios.

La tabla 3 muestra la estructura de la Parte I y sus correspondientes capítulos y la tabla 4 señala las áreas de descripción que son posibles representar de un ítem. Ambas tablas permiten que el catalogador identifique tanto el capítulo como el área de descripción que desea aplicar al momento de decidir el tipo de ítem que tienen en sus manos.

\begin{tabular}{|l|l|}
\hline Capítulo 1 & Reglas generales para la descripción \\
\hline Capítulo 2 & Libros, folletos y pliegos impresos \\
\hline Capítulo 3 & Materiales cartográficos \\
\hline Capítulo 4 & Manuscritos \\
\hline Capítulo 5 & Música \\
\hline Capítulo 6 & Grabaciones sonoras \\
\hline Capítulo 7 & Películas y videograbaciones \\
\hline Capítulo 8 & Materiales gráficos \\
\hline Capítulo 9 & Recursos electrónicos \\
\hline Capítulo 10 & Artefactos tridimensionales y realia \\
\hline Capítulo 11 & Microformas \\
\hline Capítulo 12 & Recursos continuos \\
\hline Capítulo 13 & Análisis \\
\hline
\end{tabular}

Tabla 3 Capítulos de las Reglas de Catalogación

\footnotetext{
20 Reglas de catalogación angloamericanas. $2^{2}$ ed., rev. de 2002,
} actualización de 2003, p.1.

\begin{tabular}{|l|l|}
\hline Área 1 & Título y Mención de responsabilidad \\
\hline Área 2 & Edición \\
\hline Área 3 & $\begin{array}{l}\text { Detalles específicos del material (o tipo de } \\
\text { publicación) }\end{array}$ \\
\hline Área 4 & Publicación, distribución, etc. \\
\hline Área 5 & Descripción física \\
\hline Área 6 & Serie \\
\hline Área 7 & Notas \\
\hline Área 8 & $\begin{array}{l}\text { Número normalizado y de las condiciones } \\
\text { de disponibilidad }\end{array}$ \\
\hline
\end{tabular}

\section{Tabla 4 Áreas de Descripción}

Hasta ahora se ha hablado de las partes y capítulos en que se constituyen las Reglas de Catalogación Angloamericanas y cuáles serían los capítulos a emplearse para los documentos videográficos y las grabaciones sonoras, pero haremos un espacio para fijarse en algunos de los asuntos que en los últimos dos lustros han motivado a la comunidad bibliotecaria anglosajona a repensar y replantear su código y proceso de catalogación.

Mucho se habla hoy sobre la crisis que se tuvo a finales de los noventa en la catalogación y sobre su futuro que se perfila hacia la creación de nuevos lineamientos con una estructura y organización que permitirá la descripción y representación de los objetos bibliográficos tradicionales y aquellos productos de las tecnologías de la información y el conocimiento. Los temas son muy variados: estructura lógica de las reglas, la diferencia entre contenido y contenedor, cambios en los tipos de publicaciones y los formatos múltiples, modificación en las designaciones generales del material, aparición de las estructuras de metadatos, la consolidación de los modelos conceptuales para los requerimientos funcionales de los registros bibliográficos, entre otros más.

Lo que podemos resaltar de todas esas discusiones es que la adopción de nuevos lineamientos está fomentando una serie de reflexiones en torno a teorización y prácticas comunes en la descripción bibliográfica, así como asumir una actitud de cambio para 


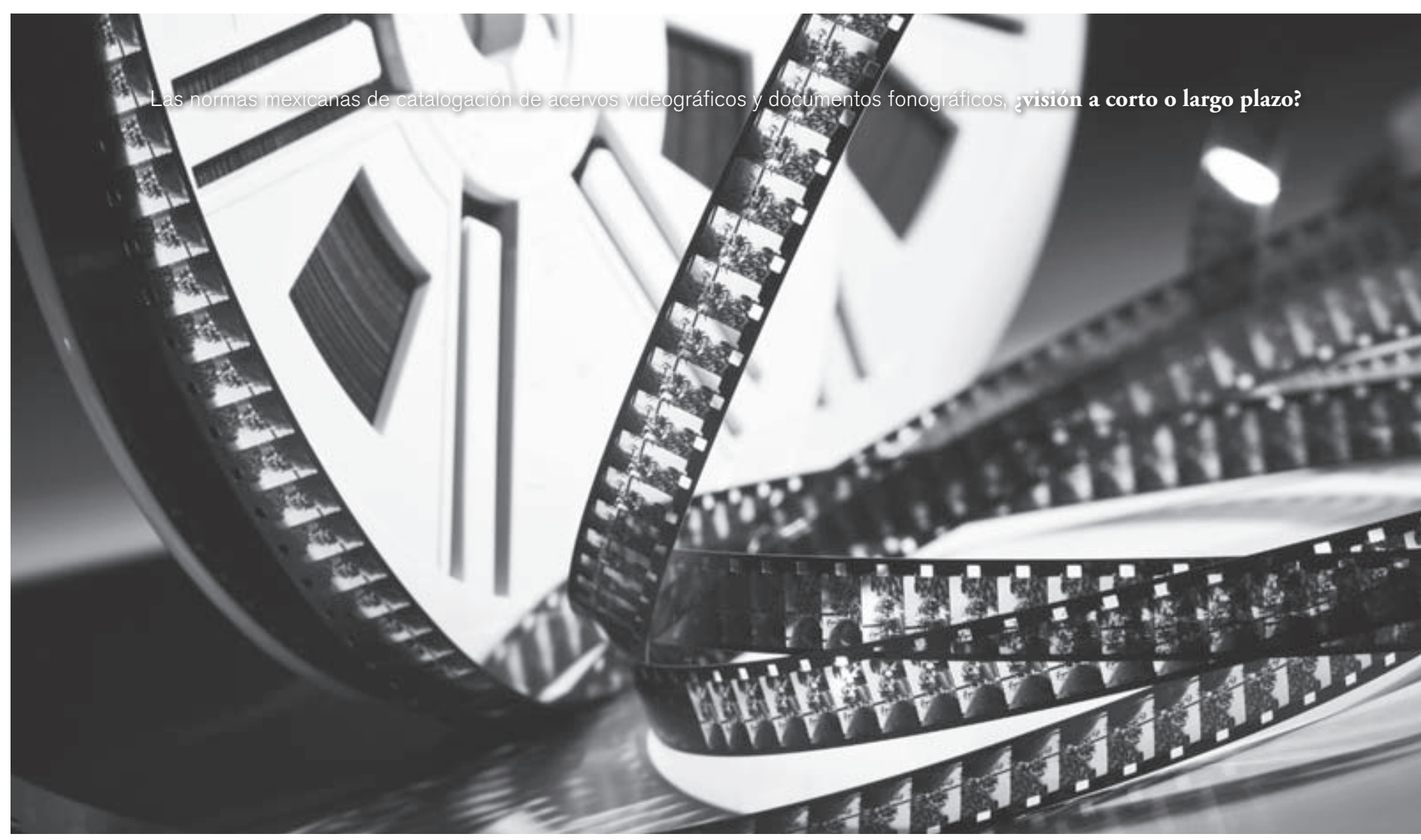

proponer proyectos alternativos que respondan a las necesidades institucionales, locales, nacionales y regionales. De ahí el interés por revisar la constitución de las normas mexicanas de catalogación de videograbaciones y grabaciones sonoras.

\section{Valoración de los principios básicos de las normas mexicanas de catalogación y las Reglas de Catalogación Angloamericanas}

El criterio que hemos tomado para realizar la valoración de los principios básicos de las normas mexicanas y las Reglas de Catalogación Angloamericanas fue, en primera conclusión, identificar los organismos responsables. Con ello respondemos a nuestra pregunta ¿Cómo se instituyen las normas mexicanas de catalogación? Desde el propio ámbito de la constitución de las normas, se observa que para su confección se creó el COTENNDOC, que es el Comité encargado de la elaboración, cambios y actualización en las disposiciones de cada norma. Este se constituye por instituciones que se encuentran en el ramo de los medios de comunicación pública y, en algunos casos, educativos. En tanto que las reglas de catalogación son elaboradas por un comité conjunto integrado por bibliotecas nacionales.

La segunda conclusión responde al cuestionamiento sobre el campo de aplicación. Las normas mexicanas señalan que su orientación es preferentemente a la catalogación de acervos nacionales audiovisuales, educativos y culturales de documentos audiovisuales y fonográficos publicados y no publicados, los cuales no persiguen un fin comercial. Nos gustaría recordar que las Reglas de Catalogación Angloamericanas están diseñadas para la elaboración de catálogos y otras listas en bibliotecas generales de cualquier tamaño, de modo que los capítulos 6 (Grabaciones) y 7 (Películas y videograbaciones) permiten la descripción de los mismos documentos tantos publicados como no publicados y, en casos particulares, las descripciones pueden ampliarse en el área 7 (Notas) con el fin de proporcionar elementos necesarios para permitir mayor especificidad a la descripción. Por ejemplo, la regla 7.7B18 Material no editado y noticieros.

Muestra de esta conclusión son los siguientes ejemplos: 


\begin{tabular}{|c|c|}
\hline Reglas & Elementos de descripción \\
\hline $\begin{array}{l}4.1 \\
4.1 .2 \\
4.1 .3 \text { y } \\
4.1 .4\end{array}$ & Sismo $1985 /$ \\
\hline $\begin{array}{l}4.2 \\
4.2 .3 \\
4.2 .3 .1 \text { y } \\
4.2 .3 .4\end{array}$ & Productor, Televisa Radio; reportero, Jacobo Zabludovsky; reportero, Guillermo Ochoa. - \\
\hline $\begin{array}{l}\text { 4.3.1, } \\
\text { 4.3.1.1, } \\
\text { 4.3.1.2, } \\
4.3 .2 \\
4.3 .2 .3 \\
4.3 .3 \text { y } \\
4.3 .3 .1\end{array}$ & Distrito Federal [México]: Televisa Radio, 19/09/1985. - \\
\hline $\begin{array}{l}\text { 4.4.1.1, } \\
4.4 .1 .2 \text { y } \\
4.4 .1 .3\end{array}$ & 1 audiofile (00:31:21:000): aleación metálica. \\
\hline 4.6.1 & Clasificado como Radio \\
\hline 4.6.1 & Número de identificación en Fonoteca Nacional: FNR0003572 \\
\hline 4.6.1 & Grabado en vivo \\
\hline 4.6.1 & $\begin{array}{l}\text { El terremoto del jueves } 19 \text { de septiembre de } 1985 \text { en la ciudad de México ha sido uno de los } \\
\text { más letales de la historia del país. Este fenómeno sismológico se suscitó a las 7:19 a. m. tiempo } \\
\text { del centro (13:19 UTC) con una magnitud de } 8.1 \text { grados en la escala de Richter, la duración } \\
\text { aproximada fue de poco más de dos minutos. }\end{array}$ \\
\hline 4.6.2 & $\begin{array}{l}\text { Jacobo Zabludovsky, Guillermo Ochoa, Lourdes Guerrero, Regina Aguirre y Guillermo Ortega } \\
\text { realizan reportes remotos de las consecuencias del sismo de } 1985 \text { en México. Describen para } \\
\text { Héctor Martínez Serrano a los estudios de la X. E. W. y el público radioescucha cómo se reciben } \\
\text { los primeros auxilios, así mismo reportan cuáles son las zonas afectadas en el Distrito Federal. }\end{array}$ \\
\hline 4.6.3 & $\begin{array}{l}\text { Se escucha el fragmento del noticiero Hoy mismo en el que María Victoria Llamas, Lourdes Gue- } \\
\text { rrero y Juan Dosal transmitían en vivo. Los periodistas Jacobo Zabludovsky y Guillermo Ochoa } \\
\text { reportan desde diferentes puntos de la ciudad de México cuáles son las pérdidas humanas y } \\
\text { materiales que dejó el sismo del } 19 \text { de septiembre de } 1985 .\end{array}$ \\
\hline 4.6 .5 & Lourdes Guerrero, locutora; Regina Aguirre, locutora; Guillermo Ortega, locutor. \\
\hline 4.6.7 & Español \\
\hline 4.7.1 & Desastre natural. Sismo. México. \\
\hline 4.7.2 & Noticias. \\
\hline
\end{tabular}


Las normas mexicanas de catalogación de acervos videográficos y documentos fonográficos, ¿̨ivisión a corto o largo plazo?

\begin{tabular}{|c|c|}
\hline Reglas & Áreas de Descripción \\
\hline $\begin{array}{l}21.1 \mathrm{C} 1 \\
6.1 \mathrm{~B} 1 \\
1.1 \mathrm{C} 1,6.1 \mathrm{C} 1\end{array}$ & Sismo 1985 [recurso electrónico] / \\
\hline $6.1 \mathrm{~F} 1$ & Producido por Televisa Radio; reportero, Jacobo Zabludovsky. - \\
\hline $\begin{array}{l}\text { 6.4C1, } \\
6.4 \mathrm{D} 1,6.4 \mathrm{~F} 1\end{array}$ & México: Televisa Radio, 1985. \\
\hline $\begin{array}{l}6.5 B 1 \\
6.5 B 2 \\
1.5 B 4 \\
6.5 C 2,6.5 C 7\end{array}$ & 1 archivo de audio (31 min., 21 seg.) : digital, estéreo \\
\hline 6.7B1 & Noticias. \\
\hline 6.7B2 & En español. \\
\hline 6.7B6 & $\begin{array}{l}\text { Jacobo Zabludovsky, Guillermo Ochoa, Lourdes Guerrero, Regina Aguirre y Guillermo Ortega, } \\
\text { reporteros; Héctor Martínez Serrano, locutor. }\end{array}$ \\
\hline 6.7B10 & Grabado en vivo. \\
\hline 6.7B14 & Apta para todo el público. \\
\hline 6.7B17 & $\begin{array}{l}\text { Sumario: El terremoto del jueves } 19 \text { de septiembre de } 1985 \text { en la ciudad de México ha sido } \\
\text { uno de los más letales de la historia del país. Este fenómeno sismológico se suscitó a las 7:19 } \\
\text { a. m. tiempo del centro (13:19 UTc) con una magnitud de } 8.1 \text { grados en la escala de Richter, la } \\
\text { duración aproximada fue de poco más de dos minutos. Jacobo Zabludovsky, Guillermo Ochoa, } \\
\text { Lourdes Guerrero, Regina Aguirre y Guillermo Ortega realizan reportes remotos de las conse- } \\
\text { cuencias del sismo de } 1985 \text { en México. Describen para Héctor Martínez Serrano a los estudios } \\
\text { de la X. E. W. y el público radioescucha cómo se reciben los primeros auxilios, así mismo repor- } \\
\text { tan cuáles son las zonas afectadas en el Distrito Federal. }\end{array}$ \\
\hline $6.7 B 18$ & $\begin{array}{l}\text { Contenido: Se escucha el fragmento del noticiero Hoy mismo en el que María Victoria Llamas, } \\
\text { Lourdes Guerrero y Juan Dosal transmitían en vivo. Los periodistas Jacobo Zabludovsky y Gui- } \\
\text { llermo Ochoa reportan desde diferentes puntos de la ciudad de México cuáles son las pérdidas } \\
\text { humanas y materiales que dejó el sismo del } 19 \text { de septiembre de } 1985 .\end{array}$ \\
\hline 6.7B19 & Para Fonoteca Nacional: FNR0003572 \\
\hline
\end{tabular}

Tabla 6 Registro con RCAA2 Capítulo 6 Grabaciones sonoras

La tercera conclusión se refiere a la Estructura o Áreas de descripción de las normas mexicanas de catalogación. Es evidente que la ausencia de una indicación en ambas normas respecto a cómo deberán distribuirse los elementos descriptivos del ítem en los registros, ya sean éstos manuales o automáticos, al catalogador le provocará confusión, abandono de la norma y, desde nuestro punto de vista, desorden en el proceso de catalogación debido a que la división de áreas cambia radicalmente en el sentido de que no es posible sistematizar el análisis, consecuentemente se desconoce cuál es el número de elementos que la componen. Además, hace falta una regla que indique qué hacer si se carece, por ejemplo, de una fuente principal de información, señalamiento que puede ser relevante cuando en los carretes, cintas y cartuchos no se cuenta con los elementos suficientes 
para su descripción. Tampoco hay una indicación o regla que establezca el nivel mínimo en la descripción ni algún señalamiento respecto a los ítems que constan de varias partes, así como una aclaración respecto a qué se entiende por serie o proyecto.

Muestra de esta conclusión son los siguientes ejemplos:

\begin{tabular}{|c|c|}
\hline Reglas & Elementos de descripción \\
\hline 5.1 & $\begin{array}{l}\text { xxv Feria Internacional del Libro del } \\
\text { Palacio de Minería. }\end{array}$ \\
\hline 5.2 & $\begin{array}{l}\text { Presentación de libro Hasta agotar la } \\
\text { existencia. }\end{array}$ \\
\hline 6.1 & $\begin{array}{l}\text { Dirección General de Televisión Universi- } \\
\text { taria (TEVE UNAM). }\end{array}$ \\
\hline 6.2 & TVIVo-24 \\
\hline 6.3 & 24 \\
\hline 6.5 & Betacam. \\
\hline 7.1 & $\begin{array}{l}\text { Presentadores: José Luis Cuevas, Carlos } \\
\text { Monsiváis y Max Rojas, moderadora: } \\
\text { Larragoiti, Josefina. }\end{array}$ \\
\hline 7.2 & TV UNAM. \\
\hline 7.3 & México. \\
\hline 8.1 & Poesía mexicana - Siglo xx. \\
\hline 8.2 & $\begin{array}{l}\text { Evento realizado en el marco de la xxv } \\
\text { Feria Internacional del Libro del Palacio } \\
\text { de Minería. }\end{array}$ \\
\hline 8.3 & $00: 60: 00$ \\
\hline 8.4 & 2004 \\
\hline 9.1 & Español. \\
\hline 10.1 & NTSC, color \\
\hline 10.3 & Estereofónico. \\
\hline
\end{tabular}

Tabla 7 Registro videográfico NMX-R-001-SCFI-2007

\begin{tabular}{|c|c|}
\hline Reglas & Áreas de descripción \\
\hline 7.1B2, 7.1.C1 & $\begin{array}{l}\text { [Presentación de libro Hasta agotar la } \\
\text { existencia] [videograbación] / }\end{array}$ \\
\hline 7.1F1 & $\begin{array}{l}\text { TV UNAM; moderadora: Larragoiti, } \\
\text { Josefina. - }\end{array}$ \\
\hline $\begin{array}{l}\text { 7.4B1, 7.4C1, } \\
\text { 7.4D1, 7.4E, 7.4F1 }\end{array}$ & $\begin{array}{l}\text { México: Dirección General de Televi- } \\
\text { sión Universitaria, } 2004 .\end{array}$ \\
\hline $\begin{array}{l}\text { 7.5B1, 7.5B2, } \\
7.5 \mathrm{C} 3,7.5 \mathrm{C} 4\end{array}$ & 1 casete $(60$ min.) : son., col. - \\
\hline 7.7B1 & Presentación de libro. \\
\hline 7.7B2 & En español. \\
\hline 7.7B3 & Título tomado del marbete. \\
\hline 7.7B6 & $\begin{array}{l}\text { Presentadores: José Luis Cuevas, } \\
\text { Carlos Monsiváis y Max Rojas. }\end{array}$ \\
\hline 7.7B10 & Estereofónico, Beta, NTSC, color. \\
\hline 7.7B10 & Copia maestra. \\
\hline 7.7B12 & $\begin{array}{l}\text { Originalmente publicada en la serie: } \\
\text { xxv Feria Internacional del Libro del } \\
\text { Palacio de Minería. }\end{array}$ \\
\hline 7.7B14 & $\begin{array}{l}\text { Público al que está destinado el ítem: } \\
\text { todo público. }\end{array}$ \\
\hline 7.7B17 & $\begin{array}{l}\text { Evento realizado en el marco de la } \\
\text { xxv Feria Internacional del Libro del } \\
\text { Palacio de Minería. }\end{array}$ \\
\hline 7.7B19 & Para TV UNAM: TVIVo-24 \\
\hline $\begin{array}{l}\text { Encabezamiento } \\
\text { de materia }\end{array}$ & Poesía mexicana - Siglo xx \\
\hline
\end{tabular}

\section{Tabla 8 Registro con RCAA2 capítulo 7 Películas y videograbaciones}

La cuarta conclusión es relativa a la concordancia con otras normas internacionales. Se observa que las normas mexicanas no tienen alguna concordancia con normas como las que tiene la Federación Internacional de Archivos de Televisión (IFTA, por sus siglas en inglés) y las normas de la Asociación Internacional de Archivos Sonoros y Audiovisuales (IASA, por sus siglas en inglés) y las que han sido objeto de estudio para este trabajo, las Reglas de Catalogación Angloamericanas, segunda edi- 
ción. Situación que deja ver, a nuestro juicio, una ruptura con las prácticas comunes de la catalogación.

De ahí que es evidente que el señalamiento sobre el alcance de las normas mexicanas sea condicionado y las posibilidades de impacto no esté más allá de las instituciones que integran el COTENNDOC. Por lo que respecta a los registros descriptivos producto de la aplicación de ambas normas, se observa que éstos no son consistentes debido a que su estructura y orden son impensados con respecto a la lógica que muestran los medios físicos (formatos) en donde se almacenan los datos descriptivos y al entendimiento tradicional de las fuentes básicas para localizar algunos elementos susceptibles de ser incluidos los registros bibliográficos, ya sean manuales o automatizados.
Finalmente, este primer acercamiento que se plantea con las normas mexicanas de catalogación, desde la perspectiva de la catalogación, ha permitido abrir varias líneas de investigación relacionadas con el tratamiento descriptivo que siguen los documentos de audio y video en las fonotecas y videotecas mexicanas, así como el alcance que se asume en las prácticas comunes en la catalogación en dichas instituciones y en aquellas pertenecientes al COTENNDOC. En suma y de acuerdo con Bianchini y Guerini ${ }^{21}$ "...las variaciones locales se permiten, por supuesto, pero en la actualidad el recurso de la elección en el ámbito local implica el riesgo de apartarse de una tradición reconocida y compartida." ${ }^{\circ}$

21 Bianchini, Carlo y Guerrini, Mauro, op. cit., p. 321.

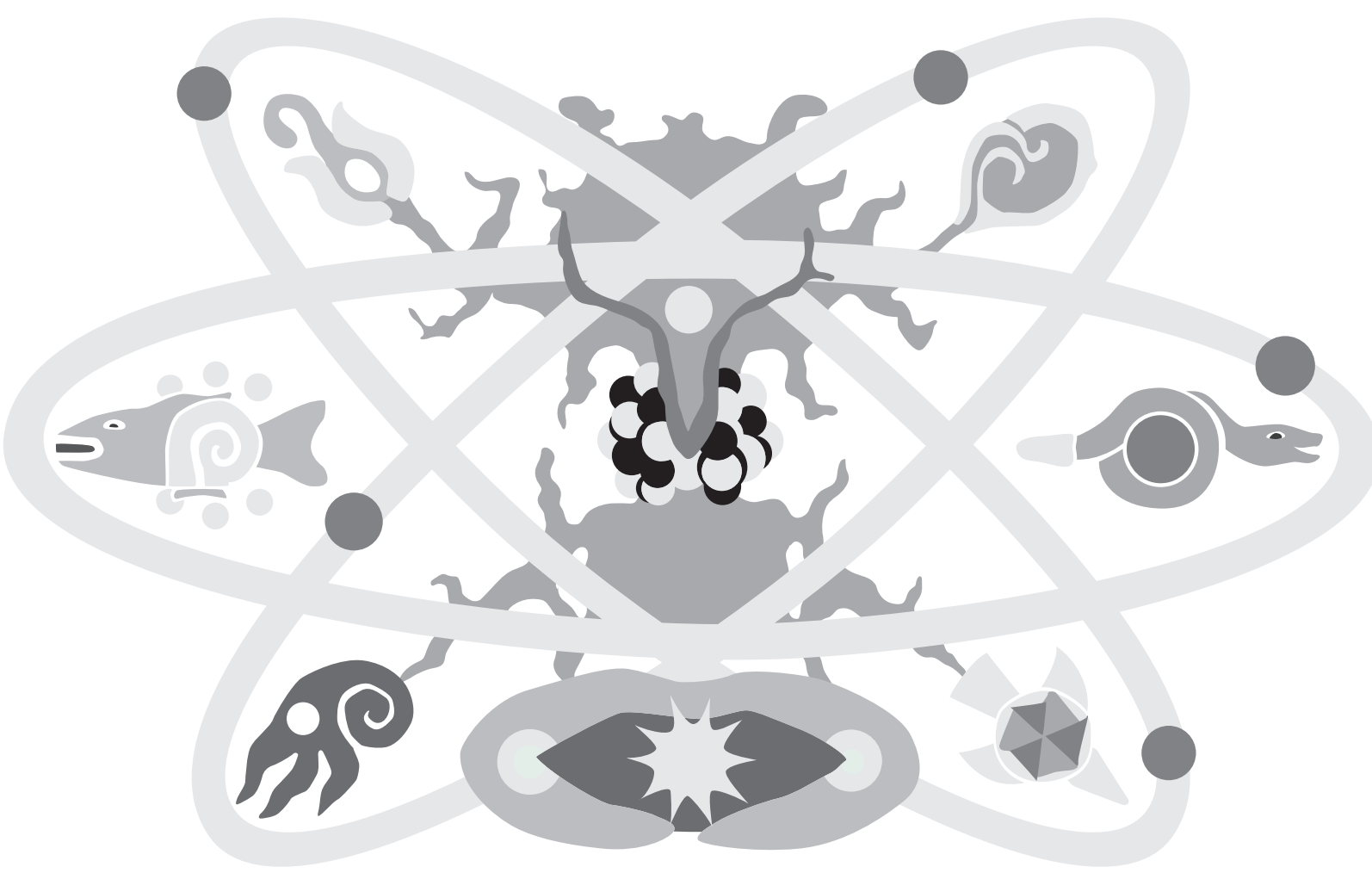




\section{Obras Consultadas}

Bianchini, Carlo y Guerrini, Mauro. Quis custodiet ipsos custodies? Observaciones sobre la relación entre FRBR, ICP, ISBD y RDA. Anales de documentación, 2009, no. 12 , p. $321-341$.

EmAnuel, Jenny. Next generation catalogs: what do they do and why should we care? Reference \& user Services Quarterly, 2009, vol. 49, no. 2, p. 117-120.

Foundations of cataloging: sourcebook. Michael Carpenter, Elaine Svenonius, editors. Littleton, Colorado: Libraries Unlimited, 1985. 276 p.

Garduño Vera, Roberto. Modelo bibliográfico basado en formatos de intercambio y en normas. México: UNAM, Centro Universitario de Investigaciones Bibliotecológicas, 1996. $224 \mathrm{p}$.

IFLA Study Group on the Functional Requirements for Bibliographic Records. Functional Requirements for Bibliographic Records [en línea]: final report. Approved by the Standing Committee of the IFLA Section on Cataloging. September 1997, as amended and corrected through February 2008. <http//www.ifla.org/VII/ s13/frbr/index.htm> [Consulta: 23 agosto 2010].

LE Bouef, Patrick. Functional requirements for bibliographic records (FRBR): hype or cure-all? New York: The Haworth Information, 2005.

Levy, David M. "Cataloging in the digital order" [en línea]. Digital libraries 95 <http://csdl.tamu.edu/ DL95/papers/levy/levy.html> [Consulta: 22 noviembre 2006]

López Guillamón, Ignacio. Evolución reciente de la catalogación. Anales de documentación. 2004, no. 7, p. 141-152.
Norma Mexicana de Catalogación de Acervos Videográficos NMX-R-001-SCFI-2007. Comité Técnico de Normalización Nacional de Documentación. México: ILCE, 2008. 60 p.

Norma mexicana NMX-R-002-SCFI-2009, documentos fonográficos [en línea]: lineamientos para su catalogación. Comité Técnico de Normalización Nacional de Documentación. México: Secretaría de Economía. 2010. <http://200.77.231.100/work/normas/nmx/2010/ nmx-r-002-scfi-2009.pdf> [Consulta: 1 julio 2010].

Oliver, Chris. Changing to RdA. Feliciter, 2007, no. 5, p. 251.

Reglas de catalogación angloamericanas. 2a ed., rev. de 2002, actualización de 2003. Bogotá: Rojas Eberhard, 2004. 1 v.

Rodríguez García, Ariel Alejandro. "El criterio del catalogador y la nueva normativa para la descripción y acceso" [en línea]. En: Memorias del vi Seminario Hispano-Mexicano de Investigación en bibliotecología y documentación. Boletín $A N A B A D$, abril-junio, 2009, no. 2, p. 247-258. <http://www.anabad.org/ boletin/index.php?id=168\&c=1>

Understanding FRBR: what it is and how it will affect our retrieval tools. Arlene Taylor, ed. Westport, Connecticut. Libraries Unlimited. 2007. 192 p.

Universidad Nacional Autónoma de México. Centro Universitario de Investigaciones Bibliotecológicas. Informe de actividades 2009-2010 [en línea]. Jaime Ríos Ortega. <http://cuib.unam.mx/f/Informe-2009-2010.pdf> [Consulta: 2 mayo 2011]. 